\title{
The ins and outs of nuclear re-export of retrogradely transported tRNAs in Saccharomyces cerevisiae
}

Jacqueline B. Pierce, Manoja B.K. Eswara and Dev Mangroo*

Department of Molecular and Cellular Biology; University of Guelph; Guelph, ON Canada

Key words: nuclear re-export of tRNA, nuclear tRNA export receptors, regulation of nuclear tRNA export, nuclear tRNA import, TOR signalling, nutrient stress

Submitted: $11 / 20 / 09$

Revised: 01/08/10

Accepted: 01/13/10

Previously published online:

www.landesbioscience.com/journals/

nucleus/article/11250

${ }^{*}$ Correspondence to:

Dev Mangroo; Email: dmangroo@uoguelph.ca
$I^{\prime}$ n Saccharomyces cerevisiae introncontaining pre-tRNAs are exported from the nucleus to the cytoplasm for removal of the introns, and the spliced tRNAs are returned to the nucleus for reasons that are not understood. The re-imported spliced tRNAs are then subjected to aminoacylation in the nucleolus to ensure that they are functional prior to re-export to the cytoplasm. Previous studies have shown that re-imported spliced tRNAs and mature tRNAs made entirely in the nucleus from intronless precursors are retained in the nucleus of $S$. cerevisiae in response to glucose, amino acid, nitrogen or inorganic phosphate deprivation. Contrary to these studies, we recently reported that starvation of $S$. cerevisiae of amino acids or nitrogen results in nuclear accumulation of re-imported spliced tRNAs, but not tRNAs made from intronless precursors. This finding suggests that separate pathways are used for nuclear export of retrogradely transported spliced tRNAs and tRNAs made from intronless pretRNAs. In addition, the data support the conclusion that the nuclear re-export pathway for retrogradely transported spliced tRNAs, but not the pathway responsible for nuclear export of tRNAs derived from intronless precursors is regulated during amino acid or nitrogen starvation. This regulation appears to occur at a step after the re-imported spliced tRNAs have undergone aminoacylation quality assurance and, in part, involves the TORC1 signalling pathway. Moreover, it was established that Utp9p is an intranuclear component that only facilitates nuclear re-export of retrogradely transported spliced tRNAs by the $\beta$-karyopherin Msn5p. Utp9p acts in concert with Utp8p, a key player in nuclear tRNA export in $S$. cerevisiae, to translocate aminoacylated re-imported spliced tRNAs from the nucleolus to Msn5p and assist with formation of the Msn5p-tRNA-Gsp1p-GTP export complex. This pathway, however, is not the only one responsible for nuclear re-export of retrogradely transported spliced tRNAs.

\section{Introduction}

Nuclear-cytoplasmic trafficking of tRNA plays a central role in key cellular processes such as regulation of protein synthesis, ${ }^{1}$ progression of cell cycle, ${ }^{2-5}$ response to nutrient availability, ${ }^{6-8}$ and development of neurodegenerative disorders. ${ }^{9,10}$ In eukaryotes, tRNAs are encoded by intronless or intron-containing tDNA genes. tRNAs from both classes of tDNA genes are synthesized in the nucleolus by PolIII-mediated transcription as precursor-tRNAs, which are processed to form functional tRNAs. ${ }^{11}$ tRNA processing involves trimming of the $5^{\prime}$ and 3 ' extensions, elaborate base modifications, addition of the nucleotides $\mathrm{C}, \mathrm{C}$ and $\mathrm{A}$ at the 3 ' ends, and removal of the introns from intron-containing pre-tRNAs. ${ }^{11}$ The process of tRNA maturation in Saccharomyces cerevisiae differs from that of mammals in both the order and the location of the maturation steps. While splicing occurs before end maturation in mammals, splicing only occurs after removal of the 5' leader and 3' trailer sequences and the addition of the $3^{\prime}$ CCA in $S$. cerevisiae. ${ }^{11}$ 


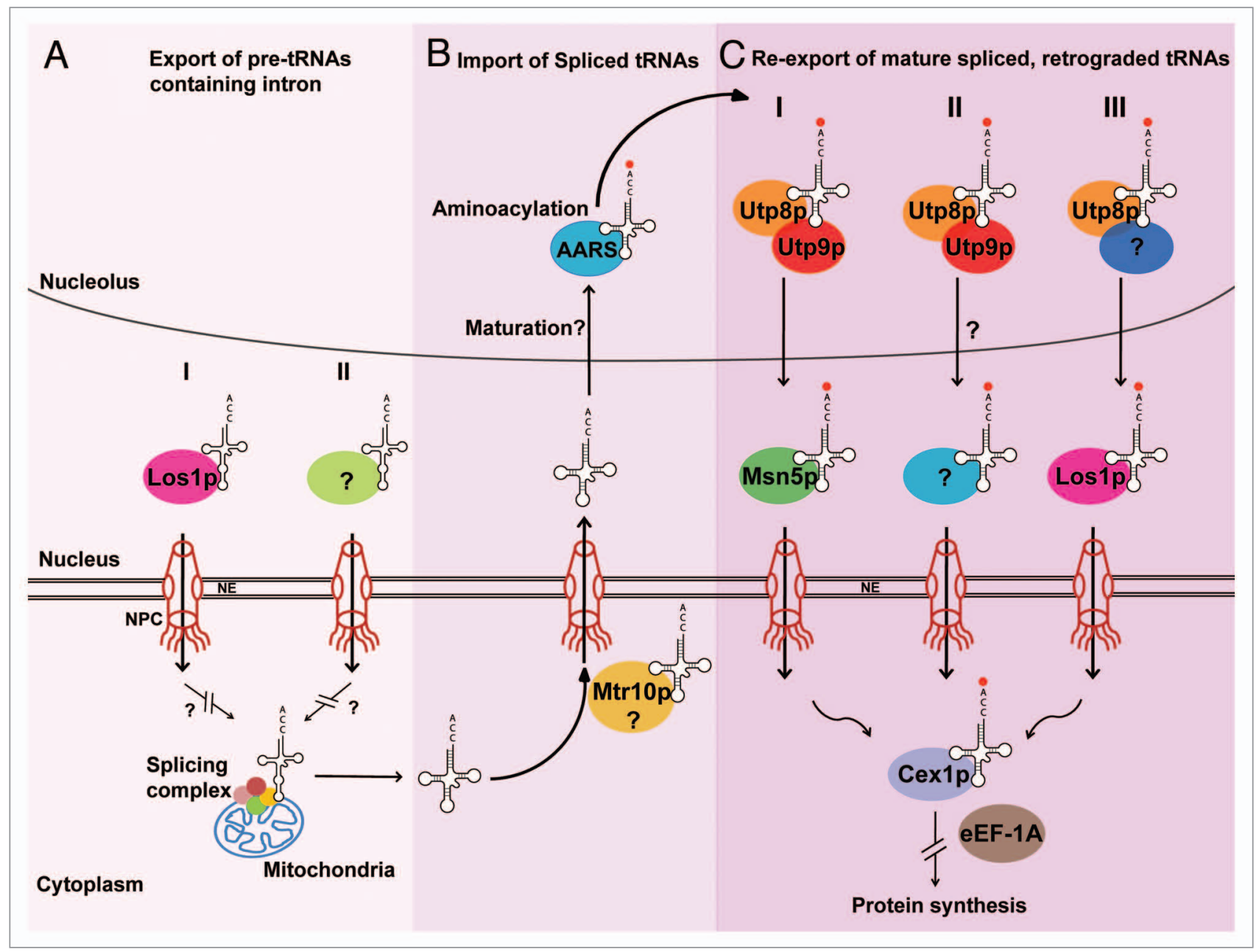

Figure 1. Pathways that facilitate nuclear export of intron-containing pre-tRNAs and nuclear re-export of retrogradely transported spliced tRNAs in S. cerevisiae. Genetic studies suggest that nuclear export of intron-containing pre-tRNAs is facilitated by Los1p (I) and an unidentified nuclear export receptor (II) (A). Upon completion of tRNA splicing in the cytoplasm, the spliced tRNAs are returned to the nucleus possibly by Mtr10p for reasons that are not understood (B). The re-imported spliced tRNAs are aminoacylated in the nucleolus and re-exported by at least three pathways named Utp9pdependent and Msn5p-dependent (I), Utp9p-dependent and Msn5p-independent (II) Utp9p-independent and Los1p-dependent (III) (C).

Moreover, in S. cerevisiae, splicing occurs in the cytoplasm, since the components of the splicing machinery are located on the surface of the mitochondria, and not in the nucleus like in mammals and plants. ${ }^{12-16}$ Hence, while the maturation of tRNAs derived from intronless pretRNAs occurs completely in the nucleus of $S$. cerevisiae, ${ }^{11}$ pre-tRNAs containing introns have to exit the nucleus to undergo splicing in the cytoplasm. Interestingly, upon completion of splicing, the spliced tRNAs are returned to the nucleus for reasons that are not understood, and are then re-exported to the cytoplasm., ${ }^{71}$ As is the case for fully processed tRNAs made in the nucleus from intronless pretRNAs, the re-imported spliced tRNAs undergo a final quality control step of aminoacylation in the nucleolus to ensure that they are functional prior to re-export to the cytoplasm for participation in protein synthesis (Fig. 1). ${ }^{18-20}$

The mechanism responsible for nuclear export of intron-containing pre-tRNAs is not entirely understood. Loslp, which is one of the nuclear tRNA export receptors in $S$. cerevisiae appears to be an export receptor in this pathway, as nuclear accumulation of unspliced pre-tRNAs was detected in a los 1 mutant strain (Fig. 1). ${ }^{21}$ Why Los1p functions as an export receptor for both mature tRNAs and introncontaining pre-tRNAs, or how Loslp discriminates between the two forms of the tRNA is not known. An unknown export receptor also facilitates nuclear export of intron-containing pre-tRNAs, since the function of Loslp is not essential (Fig. 1). ${ }^{21}$ Despite the lack of understanding of nuclear export of intron-containing pre-tRNAs, the surprising discovery that tRNA splicing occurs in the cytoplasm of $S$. cerevisiae has raised a number of questions, such as (1) Why is tRNA splicing occurring in the cytoplasm of $S$. cerevisiae and not in higher eukaryotes? (2) Can other yeasts carry out tRNA splicing in the cytoplasm, and (3) Is cytoplasmic tRNA splicing unique to $S$. cerevisiae? Investigation of these questions is likely to provide insights into the evolution of the tRNA splicing process. 


\section{Mechanism of Nuclear Import of Spliced tRNAs}

Genetic studies using mutant strains of proteins involved in nuclear tRNA export or nuclear tRNA metabolism have shown that mature tRNAs derived from introncontaining pre-tRNAs accumulate in the nucleus. 19,20,22,23 These findings led to the intriguing possibility that spliced tRNAs are imported back into the nucleus after removal of the introns. Two independent groups established that spliced tRNAs shuttle between the nucleus and cytoplasm using a heterokaryon shuttling assay and an exogenous tRNA gene containing an intron. ${ }^{7.17}$ More importantly, these data demonstrated that spliced tRNA move from the cytoplasm to the nucleus by a constitutive process, and are re-exported back to the cytoplasm. However, the details of the mechanism responsible for nuclear import of spliced tRNAs are not fully elucidated. Nevertheless, studies suggest that retrograde transport of spliced tRNAs from the cytoplasm to the nucleus occurs by two pathways, a Ranindependent, ATP-dependent pathway and a Ran-GTP/GDP-dependent pathway., ${ }^{717}$ Mtr10p, a $\beta$-karyopherin known to be involved in nuclear import of the RNA component of the yeast telomerase, appears to act as an import receptor of the Ran-dependent nuclear tRNA import pathway (Fig. 1).? However, the factors involved in the Ran-independent, ATPdependent nuclear tRNA import pathway still remain elusive.

Why spliced tRNAs are returned to the nucleus is not understood. A possibility is that they undergo further maturation and quality assurance, or just quality assurance to make sure that they are fully fit to participate in translation before they are exported back to the cytoplasm. Alternatively, the nuclear tRNA import process may be used to take improperly spliced tRNAs back to the nucleus for repair, or degradation by a nuclear tRNA degradation apparatus. ${ }^{717}$ Thus, $S$. cerevisiae may use nuclear import of tRNA as a mechanism to segregate functional cytoplasmic tRNAs from newly spliced tRNAs that have not undergone the quality assurance step to verify their functionality. It is possible, however, that nuclear import and/or re-export of spliced tRNAs play an unrealized regulatory role in a mechanism used to co-ordinate the rate of protein synthesis with the metabolic demands of the cell.

Previous studies have shown that starvation of $S$. cerevisiae for glucose, nitrogen, amino acids or inorganic phosphate results in nuclear accumulation of re-imported spliced tRNAs and mature tRNAs made entirely in the nucleus from intronless precursors. ${ }^{7,824}$ In contrast to these studies, we have shown recently that starvation of $S$. cerevisiae for amino acids or nitrogen results in nuclear accumulation of reimported spliced tRNAs, but not mature tRNAs made from intronless precursors. ${ }^{25}$ This finding led us to propose that separate pathways are used for nuclear export of retrogradely transported spliced tRNAs and mature tRNAs made from intronless precursors. Moreover, the data suggest that the nuclear re-export pathway for retrogradely transported spliced tRNAs, but not the pathway responsible for nuclear export of mature tRNAs derived from intronless precursors, is inhibited during amino acid or nitrogen starvation. This block appears to occur at a step after the re-imported spliced tRNAs have undergone aminoacylation quality assurance in the nucleolus, as retrogradely transported tRNAs have been found in the aminoacylated form in nutrient-deprived S. cerevisiae cells. ${ }^{8}$ However, the mechanism responsible for nuclear re-export of fully functional retrogradely transported spliced tRNAs to the cytoplasm is not understood.

\section{Pathways Implicated in Nuclear Export of tRNAs}

Three pathways have been implicated in nuclear tRNA export in S. cerevisiae. These pathways transport tRNAs through the nuclear pore complex (NPC) by a typical carrier-mediated process involving $\beta$-karyopherin export receptors and the yeast RanGTPase, Gsplp. Los1p, the orthologue of the mammalian Xpo-t, was the first $\beta$-karyopherin shown to be involved in nuclear tRNA export in $S$. cerevisiae. ${ }^{26-28}$ Although Msn5p, the orthologue of the mammalian minor tRNA export receptor $\mathrm{Xpo}-5,{ }^{29,30}$ is known to be involved in nuclear export and import of proteins in S. cerevisiae, it was implicated in nuclear tRNA export based on the finding that a los 1 msn 5 double mutant strain exhibited reduced efficiency of nuclear export of tRNAs made from intronless and intron-containing pre-tRNAs (data not shown). ${ }^{17}$ However, we recently provided biochemical evidence that Msn5p is an authentic tRNA export receptor. ${ }^{25}$ Despite showing reduced efficiency in nuclear tRNA export, the los 1 ms $n 5$ mutant strain is not compromised in growth or viability. ${ }^{17}$ This data combined with the observation that both Los1p and Msn5p are non-essential proteins, led to the proposal that there exists an additional nuclear tRNA export pathway involving yet unidentified receptor. Utp8p is an essential component that is common to all the nuclear tRNA export pathways in S. cerevisiae (Fig. 1). ${ }^{22}$ This protein functions as an intranuclear tRNA chaperone that collects aminoacylated tRNAs derived from intronless and intron-containing pre-tRNAs from the aminoacyl-tRNA synthetases in the nucleolus and delivers them to the nuclear export receptors of the three pathways by using a channelling mechanism. ${ }^{31,32}$ Furthermore, Utp8p facilitates the formation of the export receptortRNA-Gsplp-GTP export complex. ${ }^{31}$ Cex1p is another component that is shared by the tRNA export pathways (Fig. 1). ${ }^{33}$ Cexlp is thought to collect aminoacyltRNAs from the tRNA export receptors at the cytoplasmic face of the NPC and channels them to the translation apparatus by delivering the aminoacyl-tRNAs to the eukaryotic elongation factor eEF-1A. ${ }^{33}$ Genetic studies suggest that these pathways are responsible for nuclear export of mature tRNAs derived from intronless precursors and re-imported spliced tRNAs. ${ }^{17,18,20,22,23}$ However, we recently provided definitive genetic and biochemical evidence that the Msn5p-mediated pathway is one of the pathways responsible for nuclear re-export of retrogradely transported spliced tRNAs, and that Utp9p is a novel component involved in this pathway (Fig. 1). ${ }^{25}$ 


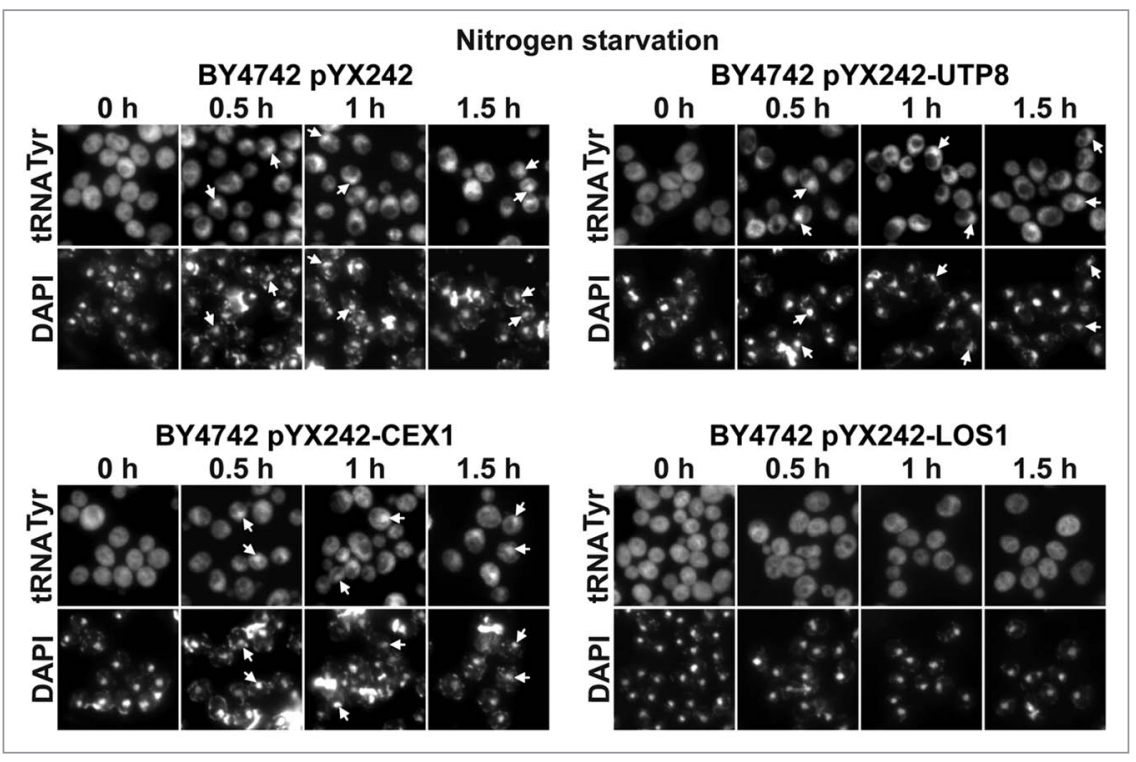

Figure 2. Los1p facilitates nuclear re-export of retrogradely transported spliced tRNAs. BY4742 pYX242, BY4742 pYX242-UTP8, BY4742 pYX242-CEX1 and BY4742 pYX242-LOS1 were starved of nitrogen in synthetic medium containing glucose and all amino acids except for leucine. At the specified times, FISH was used to monitor the cellular location of tRNA ${ }^{\text {Tyr }}$ and tRNA ${ }^{\text {Gly }}$ using Cy3labelled oligonucleotides described previously. ${ }^{25}$

\section{Pathways and Components Implicated in Nuclear Re-Export of Retrogradely Transported Spliced tRNAs}

Utp9p is an essential nucleolar protein that was shown previously to be part of a sub-complex consisting of several proteins of the U3 snoRNA-associated protein complex. ${ }^{34-36}$ This sub-complex also contains Utp8p and is involved in regulating transcription of the rRNA gene. Depletion of Utp9p was found to affect nuclear tRNA export, but not tRNA maturation and aminoacylation, or nuclear export of mRNA and ribosomal subunits. ${ }^{25}$ Utp9p binds tRNA directly in a saturable manner, but it does not function as a nuclear tRNA export receptor or an adaptor for an export receptor, as it does not shuttle between the nucleus and the cytoplasm. ${ }^{25}$ Surprisingly, loss of the function of Utp9p resulted in nuclear accumulation of mature tRNAs derived from intron-containing pre-tRNAs, but not those derived from intronless precursors. These findings led to the suggestion that Utp9p is an intranuclear component of the pathway responsible for nuclear reexport of retrogradely transported spliced tRNAs (Fig. 1). ${ }^{25}$ Moreover, Utp9p interacts in vivo and binds directly in vitro with components of the nuclear tRNA export machinery such as Utp8p, the nuclear export receptor Msn5p and Gsplp in the GTP bound form. However, unlike Utp8p, Utp9p did not interact with the nuclear tRNA export receptor Loslp or aminoacyl tRNA synthetases..$^{25}$ This finding suggests that Utp9p facilitates Msn5pmediated nuclear re-export of retrogradely transported spliced tRNAs, and functions in concert with Utp8p in translocating aminoacylated re-imported spliced tRNAs from the nucleolus to Msn5p in the nucleoplasm (Fig. 1). ${ }^{25}$ Moreover, biochemical evidence suggests that Utp9p plays a more important role than Utp8p in the formation of the Msn5p-GsplpGTP-tRNA export complex in vivo. ${ }^{25}$ However, the mechanism by which Utp9p assists with formation of the export complex is not understood. Based on in vitro protein interaction data, we surmised that both Utp8p and Utp9p bound to aminoacylated re-imported spliced tRNA interact with Msn5p; Utp9p then interacts with Gsp1p-GTP to recruit it to Msn5p, allowing Msn5p to bind the tRNA. Gsplp interacts with Utp8p to facilitate release of the tRNA and dissociation of Utp9p and Utp8p from the export complex. ${ }^{25}$
These studies demonstrated that Utp9p, Utp8p, Msn5p and Cexlp, which has been shown to co-purify with Msn5p, ${ }^{33}$ constitute a pathway that is responsible for nuclear re-export of aminoacylated retrogradely transported spliced tRNAs (Fig. 1). ${ }^{25}$ However, it is likely that other components are involved in the Utp9p-mediated nuclear tRNA re-export pathway because Msn5p and Cexlp are non-essential proteins. ${ }^{17,33}$ Since Utp9p is an essential protein and affects nuclear re-export of spliced tRNAs whereas no effect on tRNA export is detected in a msn 5 mutant strain, it is likely that Utp9p facilitates nuclear re-export of retrogradely transported spliced tRNAs by another export receptor. However, the identity of the tRNA export receptor and components of the Utp9pdependent, Msn5p-independent nuclear tRNA re-export pathway is not known (Fig. 1). Loslp is unlikely to be this receptor, as Utp9p does not interact with Los1p in vivo or in vitro. ${ }^{25}$

While the function of Loslp is not essential, studies have shown that depletion of Loslp resulted in reduced efficiency of nuclear export of tRNA ${ }^{\text {Leu }}$, tRNA ${ }^{\text {Ile }}$ and tRNA ${ }^{\mathrm{Tyr}}$, which are derived from introncontaining pre-tRNAs, and tRNA ${ }^{\mathrm{Met}}$ made from intronless precursor. ${ }^{20,23,33}$ This suggests that Loslp could also be involved in nuclear re-export of retrogradely transported spliced tRNAs. To test this possibility, we investigated whether overexpression of Los1p restores nuclear export of tRNA ${ }^{\mathrm{Tyr}}$ in $S$. cerevisiae starved of nitrogen (Fig. 2). The BY4742 wild type strain harbouring pYX242, pYX242-UTP8 or pYX242-CEX1 starved of nitrogen over a $1.5 \mathrm{~h}$ period retained tRNA ${ }^{\mathrm{Tyr}}$ in the nucleus. In contrast, nitrogen starvation of BY4742 cells overproducing Los1p did not accumulate tRNA ${ }^{\mathrm{Tyr}}$ in the nucleus, indicating that overexpression of Loslp restored nuclear re-export of $\mathrm{tRNA}^{\mathrm{Ty}}$. Western blot analysis of the same amount of total cell extract, based on actin levels, confirmed that the proteins were overexpressed (data not shown). These data suggest that Los1p can also facilitate nuclear re-export of retrogradely transported spliced tRNAs, and constitute a Utp9pindependent, Loslp-dependent nuclear tRNA re-export pathway. Whether a protein with similar function to Utp9p is 
involved in the Los1p-dependent pathway is not known. An uncharacterized pathway also facilitates nuclear re-export of retrogradely transported spliced tRNAs, as loss of the function of both Msn5p and Loslp has no effect on cell growth or viability. Thus, there appears to be at least three pathways responsible for nuclear reexport of retrogradely transported spliced tRNAs. However, the relative contribution of each pathway to the re-export of retrogradely transported spliced tRNAs is not known. The identification and characterization of the components of the unknown nuclear tRNA re-export pathway will help to address this issue.

\section{The TORC1 Signalling Pathway Regulates Nuclear Re-export of Retrogradely Transported Spliced tRNAs, but not Nuclear Export of tRNAs made from Intronless Precursors}

Nutrient stresses such as glucose, amino acid and nitrogen deprivation have been shown by others to cause nuclear accumulation of mature tRNAs derived from both intronless and intron-containing pretRNAs in S. cerevisiae. ${ }^{7,24}$ Furthermore, it was demonstrated that a regl mutant strain did not accumulate spliced tRNA ${ }^{\mathrm{Tyr}}$ in the nucleus during glucose starvation. ${ }^{8}$ In contrast, $S$. cerevisiae treated with rapamycin, which inhibits the rapamycinsensitive kinase TOR, resulted in loss of nuclear accumulation of $\mathrm{tRNA}^{\mathrm{Tyr}}$ during amino acid starvation. ${ }^{8}$ These findings suggest that the Snf1 protein kinase signalling pathway regulates nuclear import of cytoplasmic tRNAs made from both classes of pre-tRNAs in the absence of glucose whereas the TOR pathway is involved when cells are starved of amino acids. Thus, it was proposed that nuclear import of cytoplasmic tRNAs made from intronless and intron-containing pretRNAs plays a key role in nutrient-related regulation of gene expression and cell division. ${ }^{7,824}$ Contrary to previous studies, ${ }^{7,8}$ we have shown that amino acid or nitrogen starvation of $S$. cerevisiae blocks nuclear re-export of retrogradely transported spliced tRNAs, but not mature tRNAs made from intronless precursors. ${ }^{25}$ Our study, in contrast to previous reports, ${ }^{8}$

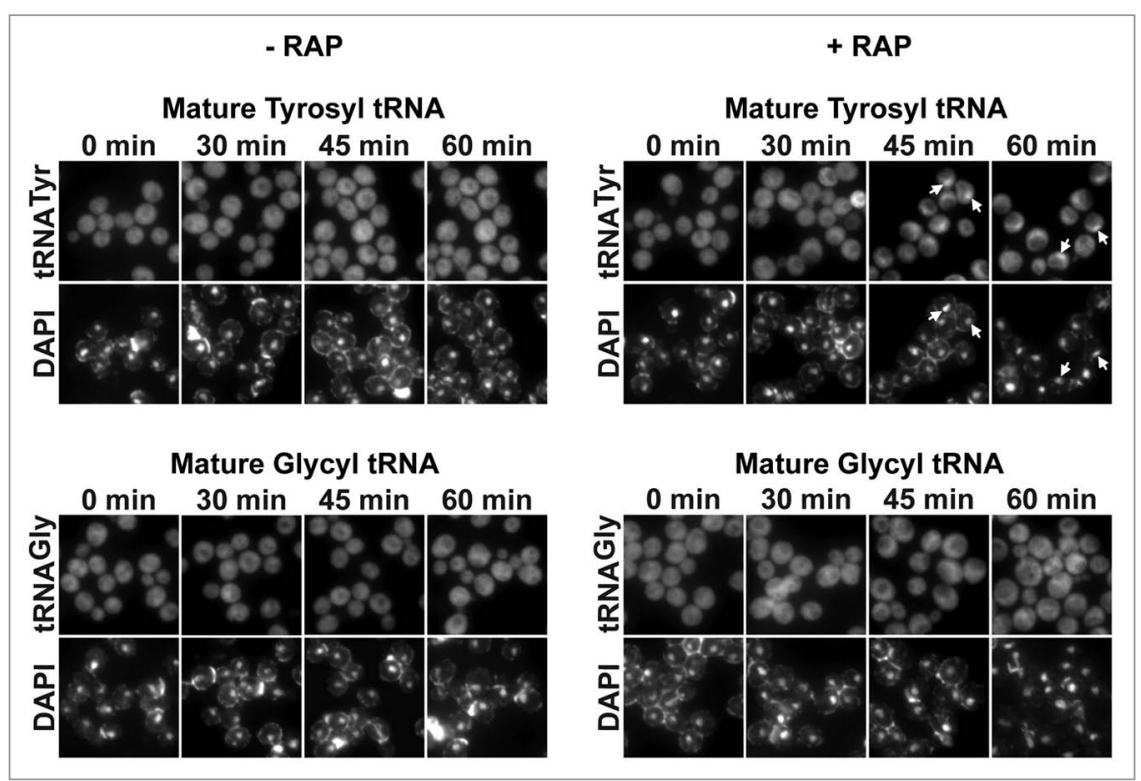

Figure 3. The TORC1 signalling pathway regulates nuclear re-export of retrogradely transported spliced tRNAs, but not nuclear export of tRNAs made from intronless pre-tRNAs. The $S$. cerevisiae TB50a strain was treated with drug delivery solution ( $90 \%$ ethanol and $10 \%$ Tween 20 ) with or without $200 \mathrm{ng} / \mathrm{ml}$ rapamycin in YPD medium for the times indicated and FISH was used to monitor the cellular location of tRNA ${ }^{\mathrm{Ty}}$ and tRNA ${ }^{\text {Gly }}$.

suggests that $S$. cerevisiae does not retrograde cytoplasmic spliced tRNAs and tRNAs made from intronless pre-tRNAs in response to amino acid or nitrogen starvation. ${ }^{25}$ Instead the data suggest that the pathway responsible for nuclear reexport of retrogradely transported spliced tRNAs, but not nuclear export of tRNAs made from intronless pre-tRNAs, is subjected to regulation in response to amino acids or nitrogen stress. ${ }^{25}$

The TORC1 signalling pathway plays a key role in controlling cell growth and division in response to a number of external stimuli, including nitrogen availability. ${ }^{37}$ Consequently, we investigated whether TORC1 is involved in regulating nuclear re-export of retrogradely transported spliced tRNAs. For this analysis, we used FISH to monitor the cellular location of mature tRNA ${ }^{\mathrm{Tyr}}$, a tRNA made from intron-containing pre-tRNA, and tRNA ${ }^{\text {Gly }}$, which is made from intronless pre-tRNA, during rapamycin treatment. Inhibition of the S. cerevisiae TORC1 by rapamycin simulates nitrogen starvation, and causes the cells to downregulate anabolic processes and upregulate catabolic and growth-inhibitory processes by a variety of mechanisms. Thus, the cells stop growth and enter a $\mathrm{G}_{0}$-like state. While previous studies have shown that rapamycin treatment does not affect nuclear tRNA export in fed cells, ${ }^{8}$ we found that exposing $S$. cerevisiae cells to rapamycin for $45 \mathrm{~min}$ to $1 \mathrm{~h}$ resulted in nuclear accumulation of mature tRNA ${ }^{\mathrm{Tyr}}$, but did not affect the distribution of mature tRNA ${ }^{\text {Gly }}$ (Fig. 3). Treatment of cells with the drug delivery solution did not affect nuclear export of tRNA ${ }^{\mathrm{Tyr}}$ and tRNA ${ }^{\mathrm{Gly}}$. In addition, tRNA ${ }^{\mathrm{Tyr}}$ was found primarily in the cytoplasm in a rapamycin-insensitive TORC1 mutant strain treated with the drug delivery solution with or without rapamycin (data not shown). These findings suggest that the TOR signalling pathway is responsible for the regulation of the nuclear re-export of retrogradely transported spliced tRNAs in S. cerevisiae cells in response to nitrogen availability.

To ascertain whether nuclear retention of re-imported spliced tRNAs during rapamycin treatment is due to a block in nuclear tRNA aminoacylation, northern blot analysis was used to monitor the aminoacylation status of tRNA ${ }^{\mathrm{Tyr}}$ in rapamycin-treated $S$. cerevisiae. We found that both nuclear and cytoplasmic tRNA $^{\mathrm{Tyr}}$ remained aminoacylated after treatment with rapamycin (Fig. 4). This result is consistent with previous studies 


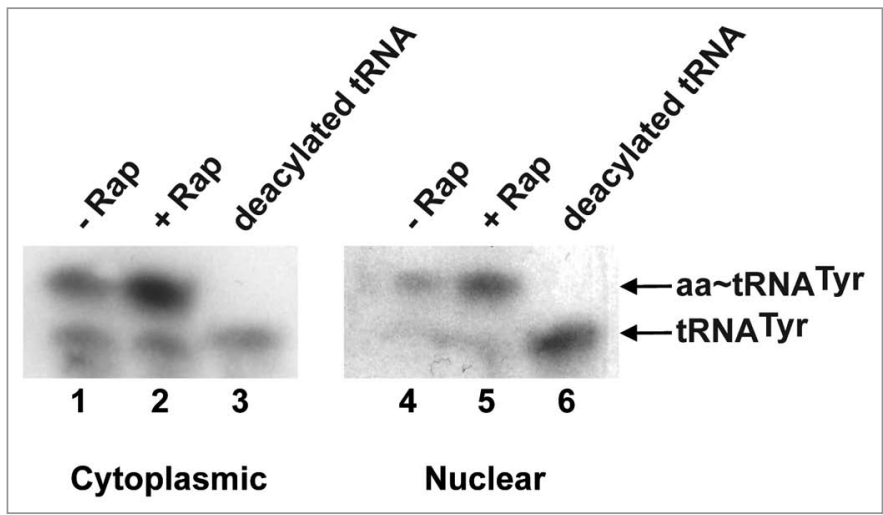

Figure 4. Rapamycin treatment does not affect nuclear and cytoplasmic aminoacylation of tRNA ${ }^{\text {Tyr }}$. The TB50a strain was incubated with drug delivery solution with or without $200 \mathrm{ng} / \mathrm{ml}$ rapamycin in YPD medium for $1 \mathrm{~h}$ and nuclear and cytoplasmic fractions were isolated. ${ }^{25}$ Total RNA from each fraction was isolated and subjected to northern blot analysis to detect tRNA $A^{\text {Tyr }} .{ }^{25}$ Deacylated tRNAs were prepared by incubating total RNA from nuclear and post-nuclear fractions in $100 \mathrm{mM}$ Tris- $\mathrm{HCl}, \mathrm{pH} 9.5$ at $37^{\circ} \mathrm{C}$ for $1 \mathrm{~h}$.

showing that retrogradely transported spliced tRNAs that accumulate in the nucleus of cells starved for amino acids are also aminoacylated. ${ }^{8}$ Moreover, we have shown that the re-export defect observed in cells depleted of Utp9p was not due to impaired nuclear tRNA aminoacylation. ${ }^{25}$ Taken together, these results indicate that rapamycin-inhibition of TOR signalling, like nitrogen deprivation, affects the nuclear re-export pathway for retrogradely transported spliced tRNAs at a step after the tRNAs have undergone aminoacylation quality assurance. However, further studies are required to understand how the TOR signalling pathway regulates nuclear re-export of retrogradely transported spliced tRNAs, and to ascertain whether TOR plays a role in regulating nuclear export of mature tRNAs made from intron-containing pre-tRNAs in metazoans.

\section{Concluding Remarks}

Nuclear re-export of retrogradely transported spliced tRNAs is facilitated by at least three pathways termed Utp9pdependent and Msn5p-dependent, Utp9pindependent and Loslp-dependent, and Utp9p-dependent and Msn5pindependent. These pathways share components and nuclear export receptors that are required for nuclear export of mature tRNAs made from intronless pre-tRNAs. However, the nuclear re-export pathways for retrogradely transported spliced tRNAs appear to be segregated from the pathways required for nuclear export of mature tRNAs made from intronless pretRNAs by utilizing novel components to select mature re-imported spliced tRNAs for re-export. Why the nuclear tRNA re-export pathways are partly separated from those that facilitate nuclear export of mature tRNAs made from intronless precursors is not understood. Another fascinating finding from our studies of nuclear tRNA export in S. cerevisiae, is the specific regulation of nuclear re-export of retrogradely transported spliced tRNAs, but not nuclear export of mature tRNAs made from intronless precursors in response to amino acids or nitrogen availability, and inhibition of the TORC1 signalling pathway using rapamycin. In addition, we have established that regulation of nuclear reexport of retrogradely transported spliced tRNAs is not due to mislocalization of Msn5p or Los1p to the cytoplasm when the cells are starved of nitrogen or amino acids or by treatment with rapamycin to inhibit TOR (unpublished data). It is possible that the activity of the nuclear tRNA export receptors, in part, is subjected to regulation, since overexpression of Loslp restored nuclear re-export of retrogradely transported $\mathrm{tRNA}^{\mathrm{Tyr}}$ when $S$. cerevisiae is starved of nitrogen. However, why nuclear re-export of retrogradely transported spliced tRNAs, but not nuclear export of tRNAs made from intronless precursors is regulated specifically by the TORC1 signalling pathway or in response to amino acid or nitrogen availability is not understood. In addition to regulation of nuclear re-export of retrogradely transported spliced tRNAs, nuclear export of intron-containing pre-tRNAs is subjected to regulation by stress based on the finding that chemical-induced DNA damage results in a block in nuclear export of intron-containing pre-tRNAs by retention of Loslp in the cytoplasm. ${ }^{4}$ Considering that intron-containing tRNAs comprise only $25 \%$ of the total tRNAs, regulation of their nuclear transport and processing in response to stress is both unanticipated and intriguing. Studies aimed at identifying and characterizing the components involved in nuclear export of introncontaining pre-tRNAs and re-export of retrogradely transported spliced tRNAs will undoubtedly provide valuable insights into the significance of the regulation of the nuclear-cytoplasmic dynamics of intron-containing tRNAs in cell growth and division.

\section{Acknowledgements}

This work was supported by operating grants from the Canadian Institutes of Health Research (CIHR) and Natural Sciences and Engineering Research Council of Canada (NSERC). J.B.P. is recipient of a NSERC Doctoral Scholarship.

\section{References}

1. Kruse C, Willkomm DK, Grunweller A, Vollbrandt T, Sommers S, Busch S, et al. Export and transport of tRNA are coupled to a multi-protein complex. Biochemical J 2000; 346:107-15.

2. White RJ. RNA polymerases I and III, growth control and cancer. Nat Rev Mol Cell Biol 2005; 6:69-78.

3. White RJ. RNA polymerase III transcription-a battleground for tumour suppressors and oncogenes. Eur J Cancer 2004; 40:21-7.

4. Ghavidel A, Kislinger T, Pogoutse O, Sopko R, Jurisica I, Emili A. Impaired tRNA nuclear export links DNA damage and cell cycle checkpoint. Cell 2007; 131:915-26.

5. Bernstein KA, Baserga SJ. The small subunit processome is required for cell cycle progression at $\mathrm{G}_{1}$. Mol Biol Cell 2004; 15:5038-46.

6. Shaheen HH, Horetsky RL, Kimball SR, Murthi A, Jefferson LS, Hopper AK. Retrograde nuclear accumulation of cytoplasmic tRNA in rat hepatoma cells in response to amino acid deprivation. Proc Natl Acad Sci USA 2007; 104:8845-50.

7. Shaheen HH, Hopper AK. Retrograde movement of tRNAs from the cytoplasm to the nucleus in Saccharomyces cerevisiae. Proc Natl Acad Sci USA 2005; 102:11290-5. 
8. Whitney ML, Hurto RL, Shaheen HH, Hopper AK. Rapid and reversible nuclear accumulation of cytoplasmic tRNA in response to nutrient availability. Mol Biol Cell 2007; 18:2678-86.

9. Schmidt WM, Kraus C, Hoger H, Hochmeister $S$, Oberndorfer F, Branks M, et al. Mutation in the Scyll gene encoding amino-terminal kinase-like protein causes a recessive form of spinocerebellar neurodegeneration. EMBO Rep 2007; 8:691-7.

10. Sone M, Uchida A, Komatsu A, Suzuki E, Ibuki I, Asada $\mathrm{M}$, et al. Loss of yata, a novel gene regulating the subcellular localization of APPL, induces deterioration of neural tissues and lifespan shortening. PLoS One 2009; 4:4466.

11. Hopper AK, Phizicky EM. tRNA transfers to the limelight. Genes Dev 2003; 17:162-80.

12. Huh WK, Falvo JV, Gerke LC, Carroll AS, Howson RW, Weissman JS, et al. Global analysis of protein localization in budding yeast. Nature 2003; 425:686-91.

13. Yoshihisa T, Yunoki-Esaki K, Ohshima C, Tanaka N, Endo T. Possibility of cytoplasmic pre-tRNA splicing: the yeast tRNA splicing endonuclease mainly localizes on the mitochondria. Mol Biol Cell 2003; 14:3266-79.

14. Yoshihisa T, Ohshima C, Yunoki-Esaki K, Endo T. Cytoplasmic splicing of tRNA in Saccharomyces cerevisiae. Genes Cells 2007; 12:285-97.

15. Paushkin SV, Patel M, Furia BS, Peltz SW, Trotta CR. Identification of a human endonuclease complex reveals a link between tRNA splicing and Pre-mRNA 3' end formation. Cell 2004; 117:311-21.

16. Englert M, Latz A, Becker D, Gimple O, Beier H, Akama K. Plant pre-tRNA splicing enzymes are targeted to multiple cellular compartments. Biochimie 2007; 89:1351-65.

17. Takano A, Endo T, Yoshihisa T. tRNA actively shuttles between the nucleus and cytosol in yeast. Science 2005; 309:140-2.

18. Steiner-Mosonyi M, Mangroo D. The nuclear tRNA aminoacylation-dependent pathway may be the principal route used to export tRNA from the nucleus in Saccharomyces cerevisiae. Biochemical J 2004; 378:809-16.
19. Sarkar S, Azad AK, Hopper AK. Nuclear tRNA aminoacylation and its role in nuclear export of endogenous tRNAs in Saccharomyces cerevisiae. Proc Natl Acad Sci USA 1999; 96:14366-71.

20. Grosshans H, Hurt E, Simos G. An aminoacylationdependent nuclear tRNA export pathway in yeast. Genes Dev 2000; 14:830-40.

21. Hurt DJ, Wang SS, Lin YH, Hopper AK. Cloning and characterization of LOS1, a Saccharomyces cer evisiae gene that affects tRNA splicing. Mol Cell Biol 1987; 7:1208-16.

22. Steiner-Mosonyi M, Leslie DM, Dehghani $H$ Aitchison JD, Mangroo D. Utp8p is an essential intranuclear component of the nuclear tRNA export machinery of Saccharomyces cerevisiae. J Biol Chem 2003; 278:32236-45.

23. Feng W, Hopper AK. A Loslp-independent pathway for nuclear export of intronless tRNAs in Saccharomyces cerevisiae. Proc Natl Acad Sci USA 2002; 99:5412-7.

24. Hurto RL, Tong AHY, Boone C, Hopper AK. Inorganic phosphate deprivation causes tRNA nuclear accumulation via retrograde transport in Saccharomyces cerevisiae. Genetics 2007; 176:841-52.

25. Eswara MB, McGuire AT, Pierce JB, Mangroo D. Utp9p Facilitates Msn5p-mediated Nuclear Re-export of Retrograded tRNAs in Saccharomyces cerevisiae. Mol Biol Cell 2009; 20: 5007-25.

26. Hellmuth K, Lau DM, Bischoff FR, Kunzler M, Hurt E, Simos G. Yeast Loslp has properties of an exportin-like nucleocytoplasmic transport factor for tRNA. Mol Cell Biol 1998; 18:6374-86.

27. Arts GJ, Fornerod M, Mattaj IW. Identification of a nuclear export receptor for tRNA. Curr Biol 1998; 8:305-14.

28. Kutay U, Lipowsky G, Izaurralde E, et al. Identification of a tRNA-specific nuclear export receptor. Mol Cell 1998; 1:359-69.

29. Bohnsack MT, Regener K, Schwappach B, Saffrich R, Paraskeva E, Hartmann E, et al. Exp5 exports eEF1A via tRNA from nuclei and synergizes with other transport pathways to confine translation to the cytoplasm. EMBO J 2002; 21:6205-15.
30. Calado A, Treichel N, Muller EC, Otto A, Kutay U. Exportin-5-mediated nuclear export of eukaryotic elongation factor $1 \mathrm{~A}$ and tRNA. EMBO J 2002 ; 21:6216-24.

31. Strub BR, Eswara MBK, Pierce JB, Mangroo D. Utp8p is a nucleolar tRNA-binding protein that forms a complex with components of the nuclear tRNA export machinery in Saccharomyces cerevisiae. Mol Biol Cell 2007; 18:3845-59.

32. McGuire AT, Keates RAB, Cook S, Mangroo D. Structural modeling identified the tRNA-binding domain of Utp8p, an essential nucleolar component of the nuclear tRNA export machinery of Saccharomyces cerevisiae. Biochem Cell Biol 2009; 87:431-43.

33. McGuire AT, Mangroo D. Cexlp is a novel cytoplasmic component of the Saccharomyces cerevisiae nuclear tRNA export machinery. EMBO J 2007; 26:288300

34. Krogan NJ, Peng WT, Cagney G, Robinson MD, Haw R, Zhong G, et al. High-definition macromolecular composition of yeast RNA-processing complexes. Mol Cell 2004; 13:225-39.

35. Gallagher JEG, Dunbar DA, Granneman S, Mitchell $\mathrm{BM}$, Osheim Y, Beyer AL, et al. RNA polymerase transcription and pre-rRNA processing are linked by specific SSU processome components. Genes Dey 2004; 18:2506-17.

36. Dragon F, Gallagher JEG, Compagnone-Post PA, Mitchell BM, Porwancher KA, Wehner KA, et al. A large nucleolar $\mathrm{U} 3$ ribonucleoprotein required for $18 \mathrm{~S}$ ribosomal RNA biogenesis. Nature 2002; 417:96770.

37. Wullschleger S, Loewith R, Hall MN. TOR signaling in growth and metabolism. Cell 2006; 124:471-84. 